\title{
Robust Market Equilibria under Uncertain Cost
}

\author{
Christian Biefel, Frauke Liers, Jan Rolfes, \\ LARs Schewe, Gregor ZÖTtL
}

\begin{abstract}
This work studies equilibrium problems under uncertainty where firms maximize their profits in a robust way when selling their output. Robust optimization plays an increasingly important role when best guaranteed objective values are to be determined, independently of the specific distributional assumptions regarding uncertainty. In particular, solutions are to be determined that are feasible regardless of how the uncertainty manifests itself within some predefined uncertainty set. Our mathematical analysis adopts the robust optimization perspective in the context of equilibrium problems. First, we present structural insights for a single-stage, nonadjustable robust setting. We then go one step further and study the more complex two-stage or adjustable case where a part of the variables can adjust to the realization of the uncertainty. We compare equilibrium outcomes with the corresponding centralized robust optimization problem where the sum of all profits are maximized. As we find, the market equilibrium for the perfectly competitive firms differs from the solution of the robust central planner, which is in stark contrast to classical results regarding the efficiency of market equilibria with perfectly competitive firms. For the different scenarios considered, we furthermore are able to determine the resulting price of anarchy. In the case of non-adjustable robustness, for fixed demand in every time step the price of anarchy is bounded whereas it is unbounded if the buyers are modeled by elastic demand functions. For the two-stage adjustable setting, we show how to compute subsidies for the firms that lead to robust welfare optimal equilibria.
\end{abstract}

Keywords. Robustness and sensitivity analysis; Equilibrium Problems; Robust Optimization; Adjustable Robustness

Corresponding AUthor. Christian Biefel - christian.biefel@fau.de

\section{INTRODUCTION}

Equilibrium problems arise whenever several agents which interact in a common context maximize their own objectives. An equilibrium then corresponds to a 'stable' situation in which no agent has an incentive to deviate from his or her optimum strategy, given the strategies of all other agents. Such tasks appear in wide contexts, for example in market interactions, in transportation, and the like. Many insights have already been obtained for the case of deterministic and risk neutral settings. In many situations however, uncertainty and risk play an essential role for the optimization problems of the different agents and the resulting equilibria. Indeed, when neglecting the influence of uncertainty, solutions may differ considerably, and so it is advisable to hedge against them.

Recently, the explicit consideration of uncertainties in equilibrium problems has obtained increased attention. Most of those contributions model the uncertainties from a stochastic optimization perspective. Stochastic optimization (Birge and Louveaux [2011]) protects against uncertainties with a certain probability and in expectation. A prominent approach, for example, consists in the

Date: February 16, 2022. 
introduction of risk functions which allows to take into account risk aversion (for the seminal work compare Artzner et al. [1999]).

A stochastic optimization approach naturally assumes that information on the underlying probability distributions is available. In many settings, however, such knowledge may not be available, or, in safety-critical situations, protection in a probabilistic sense may not be enough. Furthermore, the resulting problems are not necessarily algorithmically tractable. In such situations, a different modelling approach is appropriate that has its foundations in robust optimization.

In robust optimization (Ben-Tal et al. [2009]), the task is to ensure feasibility of a solution regardless of how uncertainties manifest themselves within predefined uncertainty sets. Among all such robust feasible solutions, a robust optimum is determined that yields best guaranteed objective values. A main challenge in this very active research area consists in modelling or reformulating the robust counterpart problem in a way that leads to algorithmical tractability. Another topic of research studies how conservative the robust solutions are, i.e., the cost of robust protection when compared to the unprotected case in which uncertainties are ignored.

In the present article, we thus present structural insights into equilibrium problems where firms in a market context maximize their profits in a robust way when selling their output. Firms optimize their investment and production decisions for several periods of time facing uncertain production costs. We first analyze the single-stage or non-adjustable robust setting, where the firms' production decisions are determined by the original output decision for all periods of time and for all realizations of cost uncertainty. We then go one step further and study the more complex two-stage or adjustable case where firms first observe the realizations of uncertainty and can then adjust their production choices that are bounded by the originally made investments. The adjustable case is typically less conservative than the one-stage problem. We derive analytical results and compare equilibrium outcomes with the corresponding robust central planner solution where the worst case sum of all profits is maximized. For our analysis we consider the case of perfectly competitive firms which act as price takers. We establish existence of the resulting robust equilibrium problems and characterize them.

As we find, the market equilibrium for the perfectly competitive firms differs from the solution of the robust central planner, which is in stark contrast to classical results regarding the efficiency of market equilibria with perfectly competitive firms, compare e.g. Joskow and Tirole [2007] or Zöttl [2010]. For the different scenarios considered we furthermore are able to determine the resulting price of anarchy (PoA), see e.g. Koutsoupias and Papadimitriou [1999] or Dubey [1986] for seminal contributions. In the case of non-adjustable robustness, for fixed demand in every time step the price of anarchy is bounded whereas it is unbounded if the buyers are modeled by elastic demand functions. In the case of adjustable robustness, we derive an approach to compute subsidies for the firms that lead to welfare optimal equilibria in the robust market. As a direct application of our results we consider a setting where the market participants aim to optimize their respective values at risk, instead of their worst-case production costs. Here, the values at risk of the market participants determine the uncertainty set for our robust problem. We summarize our findings in Table 1.

Next, we add on the brief overview of the related literature. For the case of deterministic or risk-neutral optimization, our framework has already been well studied in the context of the so called peak-load pricing literature, compare e.g. Murphy and Smeers [2005], Joskow and Tirole [2007], or Zöttl [2010]. 
More recently, a large strand of contributions has developed which considers stochastic equilibrium problems. Ehrenmann and Smeers [2011] for example characterize market equilibria in the case of risk averse firms in an adjustable peak-loadpricing framework. Ralph and Smeers [2015] include the possibility of tradable risk, which allows to reconcile risk neutral and risk averse behavior of firms. Along similar lines, results are obtained by Philpott et al. [2016]. Moreover, Schiro et al. [2016] provides general existence results of competitive equilibrium under risk aversion. Pang et al. [2017] analyze the case of strategically behaved firms. Finally, Gérard et al. [2018] show for the case of perfectly competitive firms that market equilibria for risk averse agents are not unique.

As argued above, we protect against uncertainties in a robust manner. This work thus contributes to the recent strand of literature which seeks to explicitly introduce robust optimization in the context of equilibrium problems. Equilibrium problems where players address uncertainties from a robustness perspective play an increasingly important role in the literature. For a seminal contribution in this context consider e.g. Aghassi and Bertsimas [2006]. Several recent articles consider different robust equilibrium problems under incomplete information, compare e.g. Dey and Zaman [2020], or Fanzeres et al. [2019] for robust bidding in auctions. The article Wang et al. [2012] considers a robust optimization model for a market similar to ours in continuous time, but does not include investment decisions.

Another area of research considers robust linear complementarity problems, short LCPs, which are suited to describe strictly robust equilbrium problems. In this context consider the seminal contributions by Wu et al. [2011] and Xie and Shanbhag [2016], and more recently Krebs and Schmidt [2020], or Krebs et al. [2021] which introduce a less conservative nonadjustable robust approach to LCPs. In Biefel et al. [2022], the authors consider uncertain linear complementarity under the assumption of affinely adjustable robustness and derive characterizations for conditions under which a solution exists and is unique. However, the results apply to general LCPs and do not answer questions on the market equilibria considered in the present work, for example the comparison of an equilibrium to the central planner optimum.

Finally, in recent contributions, Kramer et al. [2018] and Çelebi et al. [2021] consider robust investment and production decisions of firms in the context of electricity markets. In the setup considered, uncertainty affects only those parts of the market environment which affect all firms symmetrically (i.e. in their context common market demand is subject to uncertainties). The latter allows to obtain the equivalence of competitive market equilibrium and the corresponding system optimal benchmark. Moreover, Çelebi et al. [2021] focus on the single level-nonadjustable case, whereas we consider the (usually complex but typically less conservative) adjustable setting. In the context of our setup uncertainties are affecting firms in an asymmetric way (i.e. each firm's production cost). This breaks the equivalence of equilibrium and the corresponding system optimum, as we show, however.

The outline of this work is as follows. In Section 2, we introduce the nominal peak load pricing model. In Section 3, we relate the optimum robust objective function value of robust linear optimization problems with polyhedral uncertainty set to the similar problem but with box uncertainty sets. The results are necessary for determining the price of anarchy in later sections. In Section 4, we explain the robust peak load pricing model and use the results from Section 3 to quantify the relation between the robust central planner solution and the robust market outcome, i.e., we quantify the price of anarchy. The results are given for fixed as well as for elastic demand, see Section 5, and for the non-adjustable as well as for 
C. BIEFEL, F. LIERS, J. ROLFES, L. SCHEWE, G. ZÖTTL

TABle 1. Overview of the theoretical results (PoA: Price of Anarchy, CP: Central Planner)

\begin{tabular}{|l|l|l|}
\hline & Strict robustness & Adjustable robustness \\
\hline $\begin{array}{l}\text { Fixed demand } \\
\text { Section } 4\end{array}$ & $\begin{array}{l}\text { PoA bounded: } \\
\text { Theorem } 4.2\end{array}$ & $\begin{array}{l}\text { Strict and adjustable CP have } \\
\text { equal objective value: Theorem } 4.6 \\
\text { Examples showing PoA }>1\end{array}$ \\
\hline $\begin{array}{l}\text { Elastic demand } \\
\text { Section 5 }\end{array}$ & $\begin{array}{l}\text { PoA unbounded: } \\
\text { Theorem 5.1 }\end{array}$ & $\begin{array}{l}\text { Strict and adjustable CP have } \\
\text { equal objective value: Theorem } 5.2 \\
\text { Examples showing PoA }>1\end{array}$ \\
& Subsidies for optimal equilibrium: Theorem 5.5 \\
\hline
\end{tabular}

the adjustable problem. In Section 6, we apply the results to coherent and noncoherent risk measures, particularly to the value at risk and point out relations to data driven coherent risk measures. We end with conclusions in section 7 .

\section{Nominal Peak Load Pricing}

Following standard electricity market models, we consider a market taking place in $T$ time periods, where $\mathcal{T}=\{1, \ldots, T\}$. We assume perfect competition, i.e. all players are price takers.

On the production side of the market, there are different producers $i \in \mathcal{N}=$ $\{1, \ldots, N\}$. In order to be able to produce the considered good (for example electricity), a producer $i$ needs to build capacity $y_{i} \geq 0$, where for each newly installed capacity unit, investment $\operatorname{costs} c_{i}^{\text {inv }}$ need to be paid. In each time period $t \in \mathcal{T}$, the producers decide on their production $x_{i, t}$ that may not exceed their respective capacities. The producers have to pay their variable $\operatorname{costs} c_{i}^{\mathrm{var}}$, but receive for each produced unit some exogenously given market price $\pi_{t}$. The goal of each producer is to maximize his or her profit. Hence, the optimization problem of producer $i$ reads

$$
\begin{aligned}
\max _{x_{i}, y_{i} \geq 0} & \sum_{t \in \mathcal{T}}\left(\left(\pi_{t}-c_{i}^{\mathrm{var}}\right) x_{i, t}\right)-c_{i}^{\mathrm{inv}} y_{i} \\
\text { s.t. } & x_{i, t} \leq y_{i}, \quad t \in \mathcal{T} .
\end{aligned}
$$

We address two different cases for the demand side of the market. First, in Section 4 , we consider the case of fixed demand. Here, in each time period $t \in \mathcal{T}$ a fixed demand $d_{t}$ is given. An equilibrium $(\pi, x, y)$ is a set of prices, production choices and investments such that the optimality conditions of all players and the market clearing conditions

$$
d_{t}=\sum_{i \in \mathcal{N}} x_{i, t}, \quad t \in \mathcal{T}
$$

are fulfilled. In the following, we denote the total production in time period $t$ by $\bar{x}_{t}$, i.e. $\bar{x}_{t}=\sum_{i \in \mathcal{N}} x_{i, t}$.

The problem consisting of the optimality conditions of the producer problems (1) together with the market clearing condition (2) is equivalent to the system of necessary and sufficient optimality conditions, in this case the Karush-Kuhn-Tucker (KKT) conditions, of a linear program. This problem is called welfare optimization 
problem or central planner problem under fixed demand and reads

$$
\begin{gathered}
\max _{x, y \geq 0}-\sum_{i \in \mathcal{N}}\left(c_{i}^{\text {inv }} y_{i}+\left(\sum_{t \in \mathcal{T}} c_{i}^{\mathrm{var}} x_{i, t}\right)\right) \\
\text { s.t. } x_{i, t} \leq y_{i}, \quad i \in \mathcal{N}, t \in \mathcal{T} \\
\sum_{i \in \mathcal{N}} x_{i, t}=d_{t}, \quad t \in \mathcal{T} .
\end{gathered}
$$

This optimization problem minimizes the total cost in the market, i.e., it determines the system optimum that a centralized planning process would follow. We thus call it the central planner problem. It yields an approach to determine market clearing prizes as follows. The optimal dual variables of $(3 \mathrm{c})$ are exactly the prizes $\pi_{t}$ that lead to a market clearing equilibrium. Further, we note that (3) always has an optimal solution. Indeed, it is equivalent to a linear minimization problem that is bounded from below by zero for which thus an optimum solution is attained.

Second, in Section 5, we consider the case of elastic demand instead of fixed demand. Here, an elastic demand function $p_{t}: \mathbb{R} \rightarrow \mathbb{R}$ is given in each time period. We assume $p_{t}$ to be strictly decreasing for every $t \in \mathcal{T}$. Note that this is one of the fundamental assumptions for most microeconomic frameworks, compare e.g. Mas-Colell et al. [1995a]. With this, in each time period $t \in \mathcal{T}$, the demand side solves the problem

$$
\max _{d_{t} \geq 0} \int_{0}^{d_{t}} p_{t}(s) d s-\pi_{t} d_{t}
$$

The optimality conditions of the producers (1) and the consumers (4), together with the market clearing conditions (2) are equivalent to the KKT conditions of the welfare optimization problem under elastic demand

$$
\begin{aligned}
\max _{x, y \geq 0} & \sum_{t \in \mathcal{T}} \int_{0}^{\bar{x}_{t}} p_{t}(s) d s-\sum_{i \in \mathcal{N}}\left(c_{i}^{\mathrm{inv}} y_{i}+\left(\sum_{t \in \mathcal{T}} c_{i}^{\mathrm{var}} x_{i, t}\right)\right) \\
\text { s.t. } & x_{i, t} \leq y_{i}, \quad i \in \mathcal{N}, t \in \mathcal{T} .
\end{aligned}
$$

Since the elastic demand function is strictly decreasing, the objective function in (5) is concave. Thus, there exists an optimal solution and the KKT conditions of (5) are necessary and sufficient optimality conditions. This implies that optimal solution $\left(x^{*}, y^{*}\right)$ of $(5)$ can be extended to a market equilibrium by defining prices as $\pi_{t}=p_{t}\left(\sum_{i} x_{i, t}^{*}\right)$ for all $t \in \mathcal{T}$.

In more detail, a set of prices, investments and production choices $(\pi, y, x)$ is a market equilibrium if and only if $(y, x)$ is an optimal solution of (5). The market clearing prices are then given by $\pi_{t}=p_{t}(\bar{x})$ for all $t \in \mathcal{T}$. The vector $\bar{x}$, i.e. the total production in every time period, is unique. For more details, we refer to Crew et al. [1995] or Chapter 5 in Schewe and Schmidt [2019].

Notation. For the remainder of the paper, we define some notation. We denote the unit vectors by $e_{i}$ and the vector of all ones by $e$ (dimension clear from context). We use the notation $[n]=\{1, \ldots, n\}$. For a convex and compact set $\mathcal{C} \subset \mathbb{R}_{\geq 0}^{n}$ we define the parameter

$$
\begin{aligned}
\tau(\mathcal{C}):=\max _{x \in \mathbb{R}^{n}, \tau \in \mathbb{R}} & \\
\text { s.t. } \quad & \leq \leq x_{i}, \quad i \in[n], \\
& x \in \mathcal{C} .
\end{aligned}
$$

Thus, $\tau(\mathcal{C})$ is the largest number such that there is an $x \in \mathcal{C}$ with minimal entry equal to $\tau(\mathcal{C})$. 


\section{Robust Linear Optimization}

Our results on the different robust market models are based on auxiliary results from robust linear optimization. To the best of our knowledge, these results are new. The results of this section are also used to introduce the terminology for robust problems.

Let $A \in \mathbb{R}^{m \times n}, B \in \mathbb{R}^{m \times k}, b \in \mathbb{R}^{m}$ and let $\mathcal{X}=\left\{(x, y) \in \mathbb{R}_{\geq 0}^{n+k}: A x+B y \geq b\right\}$. For cost vectors $c \in \mathbb{R}_{\geq 0}^{n}$ and $d \in \mathbb{R}_{\geq 0}^{k}$ we consider the linear program

$$
\min _{(x, y) \in \mathcal{X}} c^{T} x+d^{T} y
$$

Let us assume that the cost vector $c$ is linearly affected by uncertainty that is modeled as $c(u)=c+\Lambda u$ for some diagonal matrix $\Lambda=\operatorname{diag}(\lambda) \in \mathbb{R}_{>0}^{n \times n}$. We assume that the uncertainty parameter $u$ lies in an arbitrary polyhedral uncertainty set of the form

$$
\mathcal{U}=\left\{u \in \mathbb{R}_{\geq 0}^{n}: P u \leq r\right\} \subseteq[0,1]^{n},
$$

with a suitably defined matrix $P$ and vector $r$ of appropriate dimension. Additionally, we assume that the projection of $\mathcal{U}$ onto any axis $\mathbb{R} e_{i}$ is $[0,1]$.

In robust optimization, one seeks to find a solution that attains the minimal guaranteed objective value among all possible realizations of the uncertainty. Such a so called robust optimum solution solves the robust counterpart of (7) which reads

$$
\operatorname{val}_{\mathrm{R}}:=\min _{(x, y) \in \mathcal{X}} c^{T} x+d^{T} y+\max _{u \in \mathcal{U}}(\Lambda u)^{T} x .
$$

Instead of computing an optimal robust solution by solving (8), one could also approximate the robust counterpart by replacing the uncertainty set with the box $\mathcal{B}=\Lambda[0,1]^{n}$. We obtain the problem

$$
\operatorname{val}_{\tilde{\mathcal{B}}}:=\min _{(x, y) \in \mathcal{X}}(c+\lambda)^{T} x+d^{T} y .
$$

For an optimal solution of $(9),\left(x^{*}, y^{*}\right)$, we define the objective value for the worstcase realization of the uncertainty

$$
\operatorname{val}_{\mathcal{B}}:=c^{T} x^{*}+d^{T} y^{*}+\max _{u \in \mathcal{U}}(\Lambda u)^{T} x^{*} .
$$

We note that $\operatorname{val}_{\mathcal{B}}$ depends on the choice of $x^{*}$. However, we always have $\operatorname{val}_{\mathrm{R}} \leq \operatorname{val}_{\mathcal{B}} \leq \operatorname{val}_{\tilde{\mathcal{B}}}$

We are interested in the quality of this approximation, i.e. the gap between $\mathrm{val}_{\mathcal{B}}$ and $\mathrm{val}_{\mathrm{R}}$. In the following theorem we obtain a bound on this gap which will be used in the following section to compare robust market equilibria with central planner solutions.

Theorem 3.1. The inequality

$$
\operatorname{val}_{\mathcal{B}} \leq \frac{1}{\tau(\mathcal{U})} \operatorname{val}_{R}
$$

holds.

Proof. First, we note that $0<\tau(\mathcal{U}) \leq 1$, where the strict inequality is due to convexity of the uncertainty set $\mathcal{U} \subseteq[0,1]^{n}$ and the assumption that the projectios of $\mathcal{U}$ onto any axis $\mathbb{R} e_{i}$ is $[0,1]$. Dualizing the inner maximization problem that 
determines the worst-case uncertainty realization in (8), yields the linear program

$$
\begin{gathered}
\operatorname{val}_{\mathrm{R}}=\min _{x, y, z} c^{T} x+d^{T} y+r^{T} z \\
\text { s.t. } A x+B y \geq b, \\
\\
P^{T} r \geq \Lambda x, \\
x, y, z \geq 0 .
\end{gathered}
$$

Its dual problem reads

$$
\begin{aligned}
\text { val }_{\mathrm{R}}=\max _{\theta, u \geq 0} & b^{T} \theta \\
\text { s.t. } & A^{T} \theta \leq c+\Lambda u, \\
& B^{T} \theta \leq d, \\
& P u \leq r .
\end{aligned}
$$

On the other hand, the dual problem of (9) is given by

$$
\begin{aligned}
\operatorname{val}_{\tilde{\mathcal{B}}}=\max _{\theta \geq 0} & b^{T} \theta \\
\text { s.t. } & A^{T} \theta \leq c+\lambda, \\
& B^{T} \theta \leq d .
\end{aligned}
$$

Let $\theta^{*}$ be an optimal solution of $(12)$ and let $\left(u^{*}, \tau(\mathcal{U})\right)$ be an optimal solution of (6) for $\mathcal{C}=\mathcal{U}$. Then,

$$
A^{T}\left(\tau(\mathcal{U}) \theta^{*}\right) \leq \tau(\mathcal{U})(c+\lambda) \leq c+\tau(\mathcal{U}) \lambda \leq c+\Lambda u^{*}
$$

and

$$
B^{T}\left(\tau(\mathcal{U}) \theta^{*}\right) \leq \tau(\mathcal{U}) d \leq d
$$

Thus, $\left(\tau(\mathcal{U}) \theta^{*}, u^{*}\right)$ is feasible for $(11)$. Hence,

$$
\operatorname{val}_{\mathrm{R}} \geq b^{T}\left(\tau(\mathcal{U}) \theta^{*}\right)=\tau(\mathcal{U}) b^{T} \theta^{*}=\tau(\mathcal{U}) \operatorname{val}_{\tilde{\mathcal{B}}} \geq \tau(\mathcal{U}) \operatorname{val}_{\mathcal{B}}
$$

Remark 3.2. Since any convex body that lies in the $[0,1]^{n}$ box can be approximated with a polytope arbitrarily well, this inequality also holds true for closed and convex but non-polyhedral uncertainty sets. In detail, let $\left(u^{*}, \tau(\mathcal{U})\right)$ be an optimal solution of (6) for $\mathcal{C}=\mathcal{U}$. Let $\mathcal{U}^{\text {in }}=\operatorname{conv}\left\{0, u^{*}\right\} \subset \mathcal{U}$ and let $\mathcal{U}^{\text {out }} \subset[0,1]^{n}$ be a polyhedral outer approximation of $\mathcal{U}$ with $u^{*} \in \mathcal{U}^{\text {out }}$. By replacing $\mathcal{U}$ with $\mathcal{U}^{\text {in }}$ in the computation of $\operatorname{val}_{R}$ and with $\mathcal{U}^{\text {out }}$ in the computation of $\operatorname{val}_{\mathcal{B}}$, one can conduct the above proof in a similar fashion.

In addition to comparing market equilibria with central planner solutions, we will also compare different robust optimization techniques applied to the central planner. A, in general, less conservative model for robust optimization is the adjustable robustness where some of the variables are so called wait-and-see variables that may be chosen after the uncertainty is revealed. In our case, we assume that $x$ may be chosen after the uncertainty $u$ is revealed. The adjustable robust counterpart of the uncertain problem then can be stated as

$$
\begin{gathered}
\operatorname{val}_{\mathrm{AR}}:=\min _{y \geq 0} \max _{u \in \mathcal{U}} \min _{x \geq 0}(c+\Lambda u)^{T} x+d^{T} y \\
\text { s.t. } A x+B y \geq b
\end{gathered}
$$

In (8) and (13), only the objective functions are uncertain. Thus, Theorem 14.2.4 in Ben-Tal et al. [2009] directly yields the following 
Theorem 3.3. Let $\mathcal{X}$ be a convex polytope. The equality

$$
\operatorname{val}_{R}=\operatorname{val}_{A R}
$$

holds.

Therefore, in our case adjustable robustness does not reduce the price of robustness of the robust solution under the mild assumption that $\mathcal{X}$ is compact. In words, this result says that it makes no difference whether first one has to choose $x$ and $y$ anticipating the worst case realization of the uncertainty, or whether one is allowed to adapt the decisions $x$ after the uncertainty manifests itself. In particular, the second approach does not improve the robust solution values. We slightly extend this result so that we will be able to apply it in Section 5. To this end, we let $g: \mathbb{R}^{n} \rightarrow \mathbb{R}$ be a convex and deterministic function, which we add to the objective function of the uncertain problem (7). The robust counterpart then reads

$$
\begin{gathered}
\text { cval }_{\mathrm{R}}:=\min _{(x, y) \geq 0} g(x)+c^{T} x+d^{T} y+\max _{u \in \mathcal{U}}(\Lambda u)^{T} x \\
\text { s.t. } A x+B y \geq b
\end{gathered}
$$

and the adjustable robust counterpart is given by

$$
\begin{gathered}
\operatorname{cval}_{\mathrm{AR}}:=\min _{y \geq 0} \max _{u \in \mathcal{U}} \min _{x \geq 0} g(x)+(c+\Lambda u)^{T} x+d^{T} y \\
\text { s.t. } A x+B y \geq b
\end{gathered}
$$

For this setting we now obtain an analogous result to Theorem 3.3.

Theorem 3.4. Let $\mathcal{X}$ be a convex polytope. The equality

$$
\operatorname{cval}_{R}=\operatorname{cval}_{A R}
$$

holds.

Proof. We adapt the proof of Theorem 14.2.4 in Ben-Tal et al. [2009] to our setting with nonlinear objective function. It is clear, that $\mathrm{cval}_{\mathrm{R}} \geq \mathrm{cval}_{\mathrm{AR}}$ holds.

It remains to show that $\mathrm{cval}_{\mathrm{R}} \leq \mathrm{cval}_{\mathrm{AR}}$ holds as well. Without loss of generality we may assume that all variables are adjustable in the adjustable robust solution, i.e. the dimension $k$ of $y$ is zero. With this, we have $\mathcal{X}=\left\{x \in \mathbb{R}_{\geq 0}^{n}: A x \geq b\right\}$ and

$$
\begin{aligned}
\operatorname{cval}_{\mathrm{R}} & =\min \left\{t: \exists x \in \mathcal{X}: \forall u \in \mathcal{U}: c(u)^{T} x+g(x)-t \leq 0\right\}, \\
\operatorname{cval}_{\mathrm{AR}} & =\min \left\{t: \forall u \in \mathcal{U}: \exists x \in X: c(u)^{T} x+g(x)-t \leq 0\right\} .
\end{aligned}
$$

We assume for contradiction that $\mathrm{cval}_{\mathrm{R}}>\mathrm{cval}_{\mathrm{AR}}$.

There exists $\bar{t} \in \mathbb{R}$ such that $\operatorname{cval}_{\mathrm{R}}>\bar{t}>$ cval $_{\mathrm{AR}}$. Then, for every $x \in \mathcal{X}$ there exists an uncertainty realization $u_{x} \in \mathcal{U}$ such that $c\left(u_{x}\right)^{T} x+g(x)-\bar{t}>0$. Hence, for every $x \in \mathcal{X}$ there exists an $\varepsilon_{x}>0$ and a neighbourhood $U_{x} \subset \mathbb{R}^{n}$ such that

$$
\forall z \in U_{x}: c\left(u_{x}\right)^{T} z+g(z)-\bar{t} \geq \varepsilon_{x} .
$$

Since $\mathcal{X}$ is assumed to be compact, we know that there exist finitely many points $x^{1}, \ldots, x^{N} \in \mathcal{X}$ such that $\mathcal{X} \subseteq \bigcup_{j=1}^{N} U_{x^{j}}$. We set $\varepsilon=\min _{j \in[N]} \varepsilon_{x^{j}}, u_{j}=u_{x^{j}}$ and define the functions

$$
f_{j}(x):=c\left(u_{j}\right)^{T} x+g(x)-\bar{t} .
$$

Since $g$ is convex, $f_{j}, j \in[N]$, are convex as well and from (14) we obtain that

$$
\max _{j \in[N]} f_{j}(x) \geq \varepsilon>0 \quad \text { for all } x \in \mathcal{X} .
$$


From Proposition 2.18 in Tuy [1998] it follows that there exist $\lambda_{j} \geq 0, \sum_{j \in[N]} \lambda_{j}=1$ such that

$$
f(x):=\sum_{j \in[N]} \lambda_{j} f_{j}(x) \geq \varepsilon>0 \quad \text { for all } x \in \mathcal{X} .
$$

On the other hand, let $\hat{u}:=\sum_{j \in[N]} \lambda_{j} u_{j} \in \mathcal{U}$. Since $\bar{t}>\operatorname{cval}_{\mathrm{AR}}$, there exists an $\hat{x} \in \mathcal{X}$ such that

$$
c(\hat{u})^{T} \hat{x}+g(\hat{x})-\bar{t} \leq 0 .
$$

However, since $c(u)$ is affine in $u$, we have $c(\hat{u})^{T} \hat{x}+g(\hat{x})-\bar{t}=\sum_{j \in[N]} \lambda_{j} f_{j}(\bar{x})$ and thus $f(\hat{x})=\sum_{j \in[N]} \lambda_{j} f_{j}(\hat{x}) \leq 0$, contradicting (15).

In this section, we gathered some theoretical results on robust optimization. These results will be used in order to prove statements on different robust market models in the remainder of this paper.

\section{Robust Peak Load Pricing with Fixed Demand}

In the peak load pricing model, different input parameters may be uncertain, for example uncertainties in the demand. In the following, however, we consider the variable costs of the producers to be uncertain.

As a standard assumption in robust optimization, we consider the uncertainty set $\mathcal{U} \subseteq[0,1]^{N \times T}$ to be a convex polytope. The uncertain variable costs are then defined by $c_{i, t}^{\mathrm{var}}(u)=c_{i}^{\mathrm{var}}+a_{i} u_{i, t}$ with nominal values $c_{i}^{\mathrm{var}}$, the uncertainty parameter $u \in \mathcal{U}$, and some scaling factors $a_{i} \geq 0$ for all $i \in \mathcal{N}$. In order to ease the exposition, we assume that the nominal values $c_{i}^{\text {var }}$ and the worst case values $c_{i}^{\text {var }}+a_{i}$ are feasible realizations of the uncertainty set. Therefore, without loss of generality, we require $0 \in \mathcal{U}$ and that the projections of $\mathcal{U}$ on any coordinate axis is $[0,1]$, i.e. for every $i \in \mathcal{N}, t \in \mathcal{T}$ there exists a $u \in \mathcal{U}$ such that $u_{i, t}=1$. Furthermore, we assume that $\mathcal{U}$ is independent of the time period such that it can be written as a Cartesian product of the form $\mathcal{U}=\prod_{t \in \mathcal{T}} \mathcal{U}^{\prime}$ for some $\mathcal{U}^{\prime} \subseteq[0,1]^{N}$.

We start by discussing the strict robustness and the extension to adjustable robustness for fixed demand in this section.

4.1. Single Stage. In the classical strict robust approach, all variables are hereand-now decisions that have to be made before the uncertainty realizes. Hence, in the corresponding robust market problem, all producers produce according to their respective worst case variable costs. Thus, a robust producer solves the problem

$$
\begin{aligned}
\max _{x_{i}, y_{i} \geq 0} & \sum_{t \in \mathcal{T}}\left(\left(\pi_{t}-\left(c_{i}^{\mathrm{var}}+a_{i}\right)\right) x_{i, t}\right)-c_{i}^{\mathrm{inv}} y_{i} \\
\text { s.t. } & x_{i, t} \leq y_{i}, \quad t \in \mathcal{T} .
\end{aligned}
$$

Such a market with strict robust producers is equivalent to a nominal market with changed variable costs $\hat{c}_{i, t}^{\mathrm{var}}:=c_{i, t}^{\mathrm{var}}+a_{i}$ for all $i \in \mathcal{N}$ and $t \in \mathcal{T}$. As described in Section 2, we compute the equilibrium of this market problem by solving the equivalent optimization reformulation

$$
\begin{array}{cl}
\min _{x, y \geq 0} & \sum_{i \in \mathcal{N}}\left(c_{i}^{\mathrm{inv}} y_{i}+\left(\sum_{t \in \mathcal{T}}\left(c_{i}^{\mathrm{var}}+a_{i, t}\right) x_{i, t}\right)\right) \\
\text { s.t. } & x_{i, t} \leq y_{i}, \quad i \in \mathcal{N}, t \in \mathcal{T}, \\
& \sum_{i \in \mathcal{N}} x_{i, t}=d_{t}, \quad t \in \mathcal{T},
\end{array}
$$


where the optimal dual variables of $(17 \mathrm{c})$ are exactly the market clearing prices for (16). Analogously to the nominal case, (17) has an optimal solution. Let $\left(x^{*}, y^{*}\right)$ be such an optimal solution of (17), and therefore the capacity and production choices in an equilibrium of the market with robust producers. We define the worst case total cost of this equilibrium by

$$
E_{R}:=\max _{u \in \mathcal{U}} \sum_{i \in \mathcal{N}}\left(c_{i}^{\mathrm{inv}} y_{i}^{*}+\left(\sum_{t \in \mathcal{T}}\left(c_{i}^{\mathrm{var}}+a_{i} u_{i, t}\right) x_{i, t}^{*}\right)\right) .
$$

In the uncertain market, each player independently deals with his or her worst case scenario. However, it is also of interest to compare the result with that of the robust central planner problem. The solutions may differ when taking into account possible correlation between the uncertainties. The robust central planner or robust total cost minimization problem reads

$$
\begin{aligned}
C_{R}:=\min _{x, y \geq 0} & \max _{u \in \mathcal{U}} \sum_{i \in \mathcal{N}}\left(c_{i}^{\mathrm{inv}} y_{i}+\left(\sum_{t \in \mathcal{T}}\left(c_{i}^{\mathrm{var}}+a_{i} u_{i, t}\right) x_{i, t}\right)\right) \\
\text { s.t. } \quad & x_{i, t} \leq y_{i}, \quad i \in \mathcal{N}, t \in \mathcal{T}, \\
& \sum_{i \in \mathcal{N}} x_{i, t}=d_{t}, \quad t \in \mathcal{T} .
\end{aligned}
$$

We now discuss the differences between the market with robust producers and the robust central planner problem. In the special case that the all-ones vector $e$ is contained in $\mathcal{U}$, for example if the uncertainty set is a box, all prices can attain their worst case value at the same time, and the robust solution needs to protect in particular against this realization of uncertainties. Thus, in this case (18) is equivalent to (17) and we obtain the following equality.

Theorem 4.1. If $e \in \mathcal{U}$, then $E_{R}=C_{R}$.

However, the uncorrelated situation seems quite unnatural in practical situations. Thus, typically one has the situation in which the objective value $C_{R}$ of the robust central planner problem (18) is smaller than the worst case total cost in the market with robust producers $E_{R}$. It is of interest to quantify this difference. It will turn out in the following that the difference only depends on the size and structure of the chosen uncertainty set $\mathcal{U}$.

Theorem 4.2. The inequalities

$$
C_{R} \leq E_{R} \leq \frac{1}{\tau(\mathcal{U})} C_{R}
$$

hold. In particular, this bound is sharp in the sense that for any $\delta>0$ there exist instances such that

$$
E_{R} \geq \frac{1-\delta}{\tau(\mathcal{U})} C_{R}
$$

Proof. Since every feasible solution of (17) is feasible for (18), it directly follows $C_{R} \leq E_{R}$ as the objective functions of $C_{R}$ and $E_{R}$ coincide for fixed $(x, y)$. The second inequalitiy, $E_{R} \leq \frac{1}{\tau(\mathcal{U})} C_{R}$, directly follows from Theorem 3.1.

We now present an instance proving the third inequality for arbitrary $\delta \in(0,1)$. To this end, let $T=1$ and $d=d_{1}=1$. Omitting the index $t$, we define $c_{i}^{\text {inv }}=0$ for all $i \in \mathcal{N}, c_{1}^{\operatorname{var}}(u)=(1-\delta) u_{1}$, and $c_{i}^{\text {var }}(u)=u_{i}$ for all $i \in \mathcal{N} \backslash\{1\}$. Let the polyhedral uncertainty set $\mathcal{U}$ be given by

$$
\mathcal{U}=\left\{u \in \mathbb{R}_{\geq 0}^{n}: P u \leq r\right\}
$$

with $P \in \mathbb{R}^{m \times N}$ and $r \in \mathbb{R}^{m}$. Since $0 \in \mathcal{U}$, w.l.o.g. we may rescale $P$ and assume $r \in\{0,1\}^{m}$. In the game with robust producers, the unique equilibrium is given by 
$\pi=1-\delta$ and $y_{1}=x_{1}=1$. Thus, the total cost of this equilibirum is $E_{R}=1-\delta$ in the worst case.

In the following, we establish an upper bound for $C_{R}$ : Inserting the parameters, we obtain

$$
\begin{aligned}
C_{R}= & \min _{x \geq 0} \max _{u \in \mathcal{U}}(1-\delta) u_{1} x_{1}+\sum_{i=2}^{N} u_{i} x_{i} \\
\text { s.t. } & \sum_{i \in \mathcal{N}} x_{i}=1 .
\end{aligned}
$$

Dualizing the inner maximization problem

$$
\max _{u \in \mathcal{U}}(1-\delta) u_{1} x_{1}+\sum_{i=2}^{N} u_{i} x_{i}
$$

yields

$$
\begin{aligned}
\min _{z \geq 0} & \sum_{i \in[m]} r_{i} z_{i} \\
\text { s.t. } & \left(P^{T} z\right)_{1} \geq(1-\delta) x_{1} \\
& \left(P^{T} z\right)_{i} \geq x_{i}, \quad i=2, \ldots, N .
\end{aligned}
$$

Thus, it holds that

$$
\begin{aligned}
C_{R}=\min _{x, z \geq 0} & \sum_{i \in[m]} r_{i} z_{i} \\
\text { s.t. } & \left(P^{T} z\right)_{1} \geq(1-\delta) x_{1} \\
& \left(P^{T} z\right)_{i} \geq x_{i}, \quad i=2, \ldots, N, \\
& \sum_{i \in \mathcal{N}} x_{i}=1 .
\end{aligned}
$$

We now construct a feasible solution $(\tilde{x}, \tilde{z})$ for $(19)$. Let $(\tilde{u}, \tau(\mathcal{U})) \in \mathbb{R}^{N+1}$ be an optimal basic solution of $(6)$ for $\mathcal{C}=\mathcal{U}$ which can be written as

$$
\begin{aligned}
\tau(\mathcal{U})=\max _{u \geq 0, \tau} & \\
\text { s.t. } & \tau-u_{i} \leq 0, \quad i \in \mathcal{N} \\
& P u \leq r .
\end{aligned}
$$

Let, w.l.o.g., $\tilde{u}_{i}=\tau(\mathcal{U})$ for $i \in[k]$ and $\tilde{u}_{i}>\tau(\mathcal{U})$ for $i \in \mathcal{N} \backslash[k]$. Thus, the first $k$ constraints in (20b) are tight. Furthermore, as there are at least $N+1$ constraints of (20) tight in the optimal basic solution $(\tilde{u}, \tau(\mathcal{U}))$, there is a subset $J \subset[m]$ of rows of $P$ with $|J| \geq 1$ such that $P_{J,}, \tilde{u}=r_{J}$.

As $(\tilde{u}, \tau(\mathcal{U}))$ is optimal, the target vector $e_{N+1}$ is a conical combination of the tight constraints in $(\tilde{u}, \tau(\mathcal{U}))$, i.e.

$$
e_{N+1}=\sum_{i \in[k]} \mu_{i}\left(e_{N+1}-e_{i}\right)+\left[\begin{array}{c}
\left(P_{J, \cdot}\right)^{T} \\
0 \cdots 0
\end{array}\right] \lambda
$$

with $\lambda \in \mathbb{R}_{>0}^{|J|}$ and $\mu \in \mathbb{R}_{\geq 0}^{k}$.

It follows that $\tilde{x}:=\left(P_{J, \cdot}\right)^{T} \lambda \geq 0$ and especially $\tilde{x}_{i}=0$ for all $i \in \mathcal{N} \backslash[k]$. Additionally, we observe $\sum_{i \in[k]} \mu_{i}=1$ and thus we also have $\sum_{i \in \mathcal{N}} \tilde{x}_{i}=1$. We obtain

$$
\lambda^{T} r_{J}=\lambda^{T} P_{J}, \tilde{u}=\tilde{x}^{T} \tilde{u}=\sum_{i \in \mathcal{N}} \tilde{x}_{i} \tilde{u}_{i}=\sum_{i \in[k]} \tilde{x}_{i} \tilde{u}_{i}=\sum_{i \in[k]} \tilde{x}_{i} \tau(\mathcal{U})=\tau(\mathcal{U}) .
$$


We define the vector $\tilde{z} \in \mathbb{R}_{\geq 0}^{m}$ by $\tilde{z}_{i}:=\lambda_{i}$ for $i \in J$ and $\tilde{z}_{i}:=0$ for $i \in[m] \backslash J$. Since $\tilde{x}=\left(P_{J, \cdot}\right)^{T} \lambda=P^{T} \tilde{z},(\tilde{x}, \tilde{z})$ is feasible for (19).

Thus, $C_{R} \leq \sum_{i \in[m]} r_{i} \tilde{z}_{i}=\sum_{i \in J} r_{i} \tilde{z}_{i}=\lambda^{T} r_{J}=\tau(\mathcal{U})$ and we obtain

$$
\frac{E_{R}}{C_{R}} \geq \frac{1-\delta}{\tau(\mathcal{U})}
$$

concluding the proof.

Remark 4.3. In the literature, this factor between the market and the central planner is often referred to as price of anarchy. Therefore, the result of the above theorem yields an explicit quantification of the price of anarchy in our robust singlestage market model, given by $\frac{1}{\tau(\mathcal{U})}$. Thus, this price can be controlled by appropriate choice of the uncertainty set against which protection is sought.

Remark 4.4. We see that the factor between the robust market and the robust central planner depends on the parameter $\tau(\mathcal{U})$. We want to further discuss this parameter. Clearly, if $e \in \mathcal{U}$, we have $\tau(\mathcal{U})=1$. Furthermore, from the definition of $\mathcal{U}$ as Cartesian product of $\mathcal{U}^{\prime}$ for every time step, we have $\tau(\mathcal{U})=\tau\left(\mathcal{U}^{\prime}\right)$. Also, from the assumptions on $\mathcal{U}^{\prime}$ and $\mathcal{U}$ we obtain that $\tau(\mathcal{U})=\tau\left(\mathcal{U}^{\prime}\right) \geq \frac{1}{N}$. Thus, an upper bound for the gap in Theorem 4.2 is $N$.

In the example showing tightness in the proof of Theorem 4.2, we set the nominal values of the cost vectors to zero. However, in relevant applications the relative deviations are usually bounded by some factor. We model this by introducing a parameter $\rho>0$ and assume $a_{i, t} \leq \rho c_{i}$ for all $i \in \mathcal{N}$. The previous theorem can be seen as the limiting case for $\rho \rightarrow \infty$.

Theorem 4.5. Let $\mathcal{U}$ be a compact and convex uncertainty set fulfilling the assumptions. Consider the set of restricted instances for which $a_{i, t} \leq \rho c_{i}$ for all $i \in \mathcal{N}$ with some parameter $\rho>0$. Then,

$$
E_{R} \leq \frac{1+\rho}{1+\rho \tau(\mathcal{U})} C_{R}
$$

Again, for every uncertainty set $\mathcal{U}$ there are instances for which this bound is sharp.

Proof sketch. Due to the similarity with the proof of Theorem 4.2, we aim at brevity here. The bound is shown by adapting the proof of Theorem 3.1. In addition, we use the assumption that $\lambda_{i} \leq \rho c_{i}$ for all $i \in[n]$.

For showing that the bound is sharp, we construct the following instance for arbitrary $\delta \in(0,1)$. Let $T=1$ (we omit the index $t$ ) and $d=1$. We define $c_{i}^{\text {inv }}=0$ for all $i \in \mathcal{N}, c_{1}^{\operatorname{var}}(u)=1+\rho(1-\delta) u_{1}$, and $c_{i}^{\mathrm{var}}(u)=1+\rho u_{i}$ for all $i \in \mathcal{N} \backslash\{1\}$. Analogously to the proof of Theorem 4.2, we now can show that

$$
\frac{E_{R}}{C_{R}} \geq \frac{1+\rho(1-\delta)}{1+\rho \tau(\mathcal{U})}
$$

on this instance.

The previous theorems show that a robust hedging against uncertainties in the variable costs leads to a gap between the optimal robust central planner and the worst case total cost in an equilibrium with robust producers. This upper bound on this gap depends on the one hand on the relative size of the deviations from the nominal costs and on the other hand on the geometry of the uncertainty set given by $\tau(\mathcal{U})$. In Section 5.1, where we assume elastic instead of fixed demand, we will see that this gap is not bounded anymore. 
4.2. Two-Stage. In this section we analyze the more sophisticated approach of adjustable robustness. While the investment decision still has to be made before the uncertainty realizes, the allocation of the production is chosen after the uncertainty realizes. For many market situation this is a relatively natural setup, where long run capacity decisions have to be made here-and-now before the uncertainty manifests itself but short run decisions can be taken once uncertainty has unravelled to large extent. The corresponding adjustable robust central planner problem can be stated as follows.

$$
\begin{aligned}
C_{A R}:=\min _{y \geq 0} \max _{u \in \mathcal{U}} \min _{x \geq 0} \sum_{i \in \mathcal{N}}\left(c_{i}^{\mathrm{inv}} y_{i}+\left(\sum_{t \in \mathcal{T}}\left(c_{i}^{\mathrm{var}}+a_{i} u_{i, t}\right) x_{i, t}\right)\right) \\
\text { s.t. } \quad x_{i, t} \leq y_{i}, \quad i \in \mathcal{N}, t \in \mathcal{T}, \\
\sum_{i \in \mathcal{N}} x_{i, t}=d_{t}, \quad t \in \mathcal{T} .
\end{aligned}
$$

As expected from Section 3, this additional freedom of waiting with decisions until the realization of the uncertainty does not improve the objective value compared to the strict robust central planner. Theorem 3.3 is directly applicable to these robust central planner problems and yields the following.

Theorem 4.6. The optimal objective value of the robust central planner and the adjustable robust central planner are equal, i.e.

$$
C_{R}=C_{A R}
$$

Next, we move to the adjustable robust market problem where the producers act as players who take robust decisions within an adjustable framework. We assume that the producers know exogenously given price functions $\pi_{t}(u)$ before they invest. Hence, every adjustable robust player solves the problem

$$
\begin{gathered}
\max _{y_{i} \geq 0} \min _{u \in \mathcal{U}} \max _{x_{i} \geq 0} \sum_{t \in \mathcal{T}}\left(\left(\pi_{t}(u)-c_{i}^{\mathrm{var}}(u)\right) x_{i, t}\right)-c_{i}^{\mathrm{inv}} y_{i} \\
\text { s.t. } \quad x_{i, t} \leq y_{i}, \quad t \in \mathcal{T} .
\end{gathered}
$$

A triple $(\pi(\cdot), y, x(\cdot))$ is an equilibrium of this adjustable robust market, if it satisfies the optimality conditions of all adjustable robust producers (22) and the market clearing condition (2). In the nominal case, prices supporting an equilibrium are given by the dual variables of the market clearing condition of the central planner problem. However, it is not obvious how to compute equilibrium supporting price functions in this adjustable robust setting. The following examples show that the optimal dual variables of the market clearing conditions in the various robust central planner formulations do not necessarily give us market clearing price functions.

Example 4.7. Consider a market with two time periods and $N=2$ producers which have costs of

$$
c_{1}^{\mathrm{inv}}=c_{2}^{\mathrm{inv}}=1, c_{i, t}^{\mathrm{var}}=1+u_{i, t}, \text { for } i=1,2, t=1,2 .
$$

We assume an uncertainty set given by $\mathcal{U}=\left\{u \in \mathbb{R}_{>0}^{2 \times 2}: u_{1, t}+u_{2, t} \leq 1, t=1,2\right\}$ and demands of $d_{1}=1$ and $d_{2}=2$. The optimal dual variables of the market clearing conditions (17c) are $\pi_{1}=2$ and $\pi_{2}=3$. If we use these prices in the market with adjustable robust producers $(22)$, and force $y_{1}+y_{2}=2$ in order to fulfill market clearing in the second time period, the producers produce more than the demand in the first time period if $u_{1,1}<1$ and $u_{2,1}<1$. 
Example 4.8. Consider a market in one time period with fixed demand $d=2$. There are $N=2$ producers which have costs of

$$
c_{1}^{\text {inv }}=c_{2}^{\text {inv }}=1, c_{1}^{\mathrm{var}}=u_{1}, c_{1}^{\mathrm{var}}=u_{2}
$$

with the uncerainty set $\mathcal{U}=\left\{u \in \mathbb{R}_{\geq 0}^{2}: u_{1}+u_{2} \leq 1\right\}$. In the problem of robust central planner (18), we can replace this uncertainty set by its vertices $\left\{u^{1}=\right.$ $\left.(1,0), u^{2}=(0,1), u^{3}=(0,0)\right\}$ and obtain the following equivalent reformulation of the robust central planner (18):

$$
\begin{aligned}
C_{R}=\min _{x, y, \tau \geq 0} & \tau+y_{1}+y_{2} \\
\text { s.t. } & \tau \geq x_{i}, \quad i=1,2, \\
& x_{i} \leq y_{i}, \quad i=1,2, \\
& x_{1}+x_{2}=2 .
\end{aligned}
$$

The optimal dual variable of $(23 \mathrm{~d})$ is given by $\pi=\frac{3}{2}$. However, for this market price, no producer will invest in the adjustable robust market, as in the worst case the sum of investment and variable cost of each producer is 2 . Next, we consider the adjustable robust central planner.

For the same instance, the adjustable robust central planner (21) can be equivalently reformulated to

$$
\begin{aligned}
C_{A R}=\min _{x, y, \tau \geq 0} & \tau+y_{1}+y_{2} \\
\text { s.t. } & \tau \geq x_{1}\left(u^{1}\right), \\
& \tau \geq x_{2}\left(u^{2}\right), \\
& x_{i}\left(u^{j}\right) \leq y_{i}, \quad i=1,2, \quad j=1,2,3, \\
& x_{1}\left(u^{j}\right)+x_{2}\left(u^{j}\right)=2, \quad j=1,2,3 .
\end{aligned}
$$

The optimal dual variables of $(24 \mathrm{e})$ are $\pi\left(u^{1}\right)=\pi\left(u^{2}\right)=0.75$ and $\pi\left(u^{3}\right)=0$. Choosing these dual variables as prices for the market with adjustable robust producers, the producers would not invest in any capacity as the prices are too low to cover the investment costs.

These examples show that the optimal dual variables of the market clearing conditions of the equivalent reformulation of the (strict) robust market (17), the robust central planner (18), and the adjustable robust central planner (21) do not support equilibria in the market with adjustable robust producers in general. We conclude from this that we need to find a more sophisticated alternative computing prices in the adjustable robust market. We will be able to resolve this problem in Section 5.2 for the more interesting setting where demands are not fixed but given via an elastic demand function.

\section{Robust Peak Load Pricing with Elastic Demand}

In this section, we discuss strict robustness as well as adjustable robustness for the peak load model with uncertain variable costs where demand is elastic. Whereas the case of fixed, unelastic demand is typically easier to be treated technically, as seen in the previous section, most standard market models indeed rely on the assumption of elastic demand which formally results from the utility maximization of customers. For a formal treatment and the microeconomic foundation of demand functions see e.g. Mas-Colell et al. [1995b].

We make the same assumptions on the uncertainty set $\mathcal{U}$ as in Section 4 . 
5.1. Single Stage. As in the case of fixed demand, we first consider the strict robust approach. Again, all decisions have to be made before the realizations of the uncertainty are revealed. We assume, that in the market only the producers take care of the uncertainty, the demand side is unchanged to the nominal case. Therefore, for exogenously given market prices $\pi_{t}$, each producer solves the same problem as in the case of fixed demand, i.e. the problem

$$
\begin{aligned}
\max _{x_{i}, y_{i} \geq 0} & \sum_{t \in \mathcal{T}}\left(\left(\pi_{t}-\left(c_{i}^{\mathrm{var}}+a_{i}\right)\right) x_{i, t}\right)-c_{i}^{\text {inv }} y_{i} \\
\text { s.t. } & x_{i, t} \leq y_{i}, \quad t \in \mathcal{T}
\end{aligned}
$$

maximizing their own profit in the worst case. As in the case of fixed demand, this market with strict robust producers is equivalent to a nominal market with modified variable $\operatorname{costs} c_{i, t}:=c_{i, t}^{\mathrm{var}}+a_{i}$. We can compute the market prices that lead to an equilibrium by solving the equivalent optimization reformulation

$$
\begin{aligned}
& \max _{x, y \geq 0} \sum_{t \in \mathcal{T}} \int_{0}^{\bar{x}_{t}} p_{t}(s) d s-\sum_{i \in \mathcal{N}}\left(c_{i}^{\mathrm{inv}} y_{i}+\left(\sum_{t \in \mathcal{T}}\left(c_{i}^{\mathrm{var}}+a_{i, t}\right) x_{i, t}\right)\right) \\
& \text { s.t. } x_{i, t} \leq y_{i}, \quad i \in \mathcal{N}, t \in \mathcal{T} .
\end{aligned}
$$

For an optimal solution $\left(x^{*}, y^{*}\right)$ of $(25)$, which exists due to the same reasons as in the nominal case, we define

$$
E_{R}^{\prime}:=\min _{u \in \mathcal{U}} \sum_{t \in \mathcal{T}} \int_{0}^{\bar{x}_{t}^{*}} p_{t}(s) d s-\sum_{i \in \mathcal{N}}\left(c_{i}^{\mathrm{inv}} y_{i}^{*}+\left(\sum_{t \in \mathcal{T}}\left(c_{i}^{\mathrm{var}}+a_{i} u_{i, t}\right) x_{i, t}^{*}\right)\right) .
$$

On the other hand, the robust central planner problem is given by

$$
\begin{aligned}
C_{R}^{\prime}:=\max _{x, y \geq 0} \min _{u \in \mathcal{U}} \sum_{t \in \mathcal{T}} \int_{0}^{\bar{x}_{t}} p_{t}(s) d s-\sum_{i \in \mathcal{N}}\left(c_{i}^{\mathrm{inv}} y_{i}+\left(\sum_{t \in \mathcal{T}}\left(c_{i}^{\mathrm{var}}+a_{i} u_{i, t}\right) x_{i, t}\right)\right) \\
\text { s.t. } x_{i, t} \leq y_{i}, \quad i \in \mathcal{N}, t \in \mathcal{T} .
\end{aligned}
$$

It is clear, that $C_{R}^{\prime} \geq E_{R}^{\prime}$ holds. In the case of fixed demand, the welfare is just the negative total cost. We have shown that the ratio of $E_{R}$ to $C_{R}$ is bounded by some parameter which can be computed directly from the uncertainty set $\mathcal{U}$. However, in this section the welfare $W_{R}^{\prime}$ is complemented by a nonlinear term reflecting the produced amount. As shown next, this leads to the unboundedness of the ratio, even in the case of affine demand function.

Theorem 5.1. For any $\eta>0$ there exist instances such that $C_{R}^{\prime}>\eta E_{R}^{\prime}$

Proof. We construct an example with the desired property. Let $\mathcal{N}=2$ and $T=1$. Furthermore, let $c_{1}^{\text {inv }}=c_{2}^{\text {inv }}=0$ and $c_{1}^{\text {var }}=u_{1}$ and $c_{2}^{\text {var }}=u_{2}+\varepsilon$ with $0<\varepsilon \ll 1$. The uncertainty set is given by $\mathcal{U}=\left\{u \in \mathbb{R}_{>0}^{2}: u_{1}+u_{2} \leq 1\right\}$.

We consider an affine demand function $p(s)=\alpha-s$. In the market with robust producers, only producer 1 produces as he is slightly cheaper in the worst case. Therefore, (25) reads

$$
\begin{aligned}
& \max _{x \geq 0} \int_{0}^{x_{1}+x_{2}}(\alpha-s) d s-x_{1}-(1+\varepsilon) x_{2} \\
& =\max _{x_{1} \geq 0} \int_{0}^{x_{1}}(\alpha-s) d s-x_{1}=\max _{x_{1} \geq 0}(\alpha-1) x_{1}-\frac{1}{2} x_{1}^{2} .
\end{aligned}
$$

For $\alpha>1$, the maximum is attained at $x_{1}=\alpha-1$. Thus,

$$
E_{R}^{\prime}=(\alpha-1)^{2}-\frac{1}{2}(\alpha-1)^{2}=\frac{(\alpha-1)^{2}}{2}
$$


for $\alpha>1$ and $E_{R}^{\prime}=0$ for $\alpha \leq 1$. However, the optimal robust welfare is computed by

$$
C_{R}^{\prime}=\max _{x \geq 0} \min _{u \in \mathcal{U}} \int_{0}^{x_{1}+x_{2}}(\alpha-s) d s-x_{1} u_{1}-x_{2} u_{2},
$$

where we omit the neglectable factor $\varepsilon$. Due to the symmetry of $\mathcal{U}$, the optimal solution is attained for $x_{1}=x_{2}$. Hence,

$$
C_{R}^{\prime}=\max _{d \geq 0} \int_{0}^{d}(\alpha-s) d s-\frac{1}{2} d=\max _{d \geq 0}\left(\alpha-\frac{1}{2}\right) d-\frac{1}{2} d^{2} .
$$

For $\alpha>0.5$, the maximum is attained at $d=\alpha-0.5$ and therefore we obtain

$$
C_{R}^{\prime}=\frac{\left(\alpha-\frac{1}{2}\right)^{2}}{2}
$$

for $\alpha>0.5$ and $C_{R}^{\prime}=0$ for $\alpha \leq 0.5$. Thus, for $\alpha>1$ we have

$$
\frac{C_{R}^{\prime}}{E_{R}^{\prime}}=\frac{\left(\alpha-\frac{1}{2}\right)^{2}}{(\alpha-1)^{2}} \text {. }
$$

For $\alpha \in(0.5,1]$, there is no production in the market, but in the optimal robust welfare solution there is still some small symmetrical production. Hence, in this interval the ratio is infinity. For $\alpha \leq 0.5$, there is no production in both settings.

5.2. Two-Stage. Again, we discuss the more sophisticated approach of adjustable robustness. The adjustable robust central planner under elastic demand can be stated analogously as in the corresponding section on fixed demand by

$$
\begin{gathered}
C_{A R}^{\prime}:=\max _{y \geq 0} \min _{u \in \mathcal{U}} \max _{x \geq 0} \sum_{t \in \mathcal{T}} \int_{0}^{\bar{x}_{t}} p_{t}(s) d s-\sum_{i \in \mathcal{N}}\left(c_{i}^{\mathrm{inv}} y_{i}+\left(\sum_{t \in \mathcal{T}}\left(c_{i}^{\mathrm{var}}+a_{i} u_{i, t}\right) x_{i, t}\right)\right) \\
\text { s.t. } x_{i, t} \leq y_{i}, \quad i \in \mathcal{N}, t \in \mathcal{T} .
\end{gathered}
$$

As in the case of fixed demand, Theorem 3.4 directly yields the following statement.

Theorem 5.2. The optimal Robust Welfare and the optimal Adjustable Robust Welfare are equal, i.e.

$$
C_{R}^{\prime}=C_{A R}^{\prime}
$$

In the adjustable robust market, we assume, that the producers know exogenously given price functions $\pi_{t}(u)$ before they invest. Hence, every adjustable robust player solves the problem

$$
\begin{gathered}
\max _{y_{i} \geq 0} \min _{u \in \mathcal{U}} \max _{x_{i} \geq 0} \sum_{t \in \mathcal{T}}\left(\left(\pi_{t}(u)-c_{i}^{\mathrm{var}}(u)\right) x_{i, t}\right)-c_{i}^{\mathrm{inv}} y_{i} \\
\text { s.t. } \quad x_{i, t} \leq y_{i}, \quad t \in \mathcal{T} .
\end{gathered}
$$

A triple $(\pi(\cdot), y, x(\cdot))$ is an equilibrium of this adjustable robust market, if it satisfies the optimality conditions of all adjustable robust producers (28) and of the consumer (4). We first show that in general there is a gap between the welfare of an equilibrium and the optimum of the adjustable robust central planner by the following example.

Example 5.3. Consider an instance with only one timestep, i.e. $T=1$, and two producers, i.e. $N=2$, and an elastic demand function given by $p(s)=5-s$. We define investment $\operatorname{costs} c_{i}^{\text {inv }}=\frac{1}{5}$ and nominal variable $\operatorname{costs} c_{i}^{\text {var }}=0$ for $i=1,2$, uncertainty set $\mathcal{U}=\operatorname{conv}\left\{(0,0),(1,0),(0,1),\left(\frac{3}{4}, \frac{3}{4}\right)\right\}$ and $a_{1}=a_{2}=4$. Again, as in 
Example 4.8, it suffices to consider the four extreme points of the scaled polytope, namely $u^{0}=(0,0), u^{1}=(4,0), u^{2}=(0,4)$, and $u^{3}=(3,3)$.

For the solution of the adjustable robust central planner (27), the worst case scenario is $u^{3}$ and the optimality condition reads $5-\left(y_{1}^{*}+y_{2}^{*}\right)-3-\frac{1}{5}=0$ which yields optimal capacities $y_{1}^{*}=y_{2}^{*}=0.9$. The optimal solutions and objective values for these capacities are given by

\begin{tabular}{c|c|c|c|c} 
& $u^{0}$ & $u^{1}$ & $u^{2}$ & $u^{3}$ \\
\hline optimal objective value & 7.02 & 3.74 & 3.74 & 1.62 \\
optimal production $x^{*}$ & $(0.9,0.9)$ & $(0.1,0.9)$ & $(0.9,0.1)$ & $(0.9,0.9)$
\end{tabular}

Thus, the adjustable robust central planner has a worst case objective value of $C_{A R}^{\prime}=1.62$.

There are no market prices $\pi^{*}(\cdot)$ that would extend the central planner solution to an equilibrium of the market with adjustable robust producers. However, for this simple example we can compute an equilibrium by hand. In fact, an equilibrium is given by $\left(\pi^{\prime}, y^{\prime}, x^{\prime}\right)$ with

$$
\begin{aligned}
& \pi^{\prime}=4.2, \\
& y^{\prime}=(0.4,0.4), \\
& x^{\prime}=(0.4,0.4),
\end{aligned}
$$

which can be verified by simple calculations. In case of scenario $u^{3}$, the welfare of this solution is given by

$$
2 \cdot 0.4+2 \cdot 0.4-\frac{1}{2} \cdot 0.8^{2}-0.2 \cdot 0.8=0.92
$$

which is smaller than $C_{A R}^{\prime}=1.62$.

The question now is, how to compute equilibrium supporting price functions in general. In the case of fixed demand, we showed in different examples that the usual approach, using the dual variables of the central planner, does not work. Similar examples can be constructed for this case of elastic demand by replacing the fixed demands in Example 4.7 and Example 4.8 by suitable elastic demand functions.

We postpone this discussion to future research and instead discuss an approach to induce a welfare optimal equilibrium. For this, we consider the idea of subsidizing the producers for their investment. In detail, additionally to the market prices $\pi_{t}(\cdot)$, each player $i \in \mathcal{N}$ receives a subsidy payment $\eta_{i}$ for building capacity. Therefore, each player solves the problem

$$
\begin{gathered}
\max _{y_{i} \geq 0} \min _{u \in \mathcal{U}} \max _{x_{i} \geq 0} \sum_{t \in \mathcal{T}}\left(\left(\pi_{t}(u)-c_{i}^{\mathrm{var}}(u)\right) x_{i, t}\right)-\left(c_{i}^{\mathrm{inv}}-\eta_{i}\right) y_{i} \\
\text { s.t. } \quad x_{i, t} \leq y_{i}, \quad t \in \mathcal{T} .
\end{gathered}
$$

We now show how to compute market prices and subsidies supporting an equilibrium equal to the solution of the robust central planner problem (26). For this, we follow an idea from O'Neill et al. [2005] for markets with integer variables. Let $u \in \mathcal{U}$ be arbitrary but fixed and let $y^{*}$ be the optimal capacities in the strict robust central planner problem (26). We consider the problem

$$
\begin{aligned}
\max _{x, y \geq 0} & \sum_{t \in \mathcal{T}} \int_{0}^{\bar{x}_{t}} p_{t}(s) d s-\sum_{i \in \mathcal{N}}\left(c_{i}^{\mathrm{inv}} y_{i}+\sum_{t \in \mathcal{T}} c_{i, t}^{\mathrm{var}}(u) x_{i, t}\right) \\
\text { s.t. } & x_{i, t} \leq y_{i}, \quad i \in \mathcal{N}, t \in \mathcal{T}, \\
& y_{i}=y_{i}^{*}, \quad i \in \mathcal{N} .
\end{aligned}
$$


The KKT-conditions of this problem are given by

$$
\begin{aligned}
p_{t}\left(\bar{x}_{t}\right)-c_{i, t}^{\mathrm{var}}(u)-\mu_{i, t}+\phi_{i, t} & =0, & & i \in \mathcal{N}, t \in \mathcal{T}, \\
-c_{i}^{\mathrm{inv}}+\sum_{t} \mu_{i, t}+\chi_{i}+\eta_{i} & =0, & & i \in \mathcal{N}, \\
x_{i, t}-y_{i} & \leq 0, & & i \in \mathcal{N}, t \in \mathcal{T}, \\
y_{i}-y_{i}^{*} & =0, & & i \in \mathcal{N}, \\
\mu_{i, t}\left(x_{i, t}-y_{i}\right) & =0, & & i \in \mathcal{N}, t \in \mathcal{T}, \\
\phi_{i, t} x_{i, t} & =0, & & i \in \mathcal{N}, t \in \mathcal{T}, \\
\chi_{i} y_{i} & =0, & & i \in \mathcal{N}, t \in \mathcal{T}, \\
x, y, \mu, \phi, \chi & \geq 0 . & &
\end{aligned}
$$

We define the price functions by $\pi_{t}^{*}(u)=p_{t}\left(\bar{x}_{t}^{*}(u)\right)$, where $x^{*}(u)$ is the optimal solution to (30) for the respective $u$. For every $i \in \mathcal{N}$ we compute the subsidy payment by

$$
\begin{aligned}
\eta_{i}^{*}=\max _{x, y, \mu, \phi, \chi, \eta} & \eta_{i} \\
\text { s.t. } & \exists u \in \mathcal{U}:(x, y, \mu, \phi, \chi, \eta) \text { solve }(31)
\end{aligned}
$$

Lemma 5.4. Let $x^{*}(u)$ be the optimal solution of (30) for given $u \in \mathcal{U}$. Then, for every producer $i$ with $y_{i}^{*}>0$,

$$
\eta_{i}^{*}=c_{i}^{i n v}+\max _{u \in \mathcal{U}}\left\{\sum_{t \in \mathcal{T}_{i, u}}\left(c_{i, t}^{v a r}(u)-p_{t}\left(\bar{x}_{t}^{*}(u)\right)\right)\right\}
$$

with $\mathcal{T}_{i, u}\left(x^{*}\right)=\left\{t \in \mathcal{T}: x_{i, t}^{*}(u)>0\right\}$.

Proof. From (31b) and (31g) we have $\eta_{i}=c_{i}^{\text {inv }}-\sum_{t} \mu_{i, t}$. Condition (31a) gives us

$$
\mu_{i, t}=p_{t}\left(\bar{x}_{t}\right)-c_{i, t}^{\mathrm{var}}(u)+\phi_{i, t}, \quad i \in \mathcal{N}, t \in \mathcal{T} .
$$

Thus, (32) is equivalent to

$$
\begin{aligned}
c_{i}^{\mathrm{inv}}-\min _{u, x, y, \mu, \phi, \chi, \eta} & \sum_{t \in \mathcal{T}}\left(p_{t}\left(\bar{x}_{t}\right)-c_{i, t}^{\mathrm{var}}(u)+\phi_{i, t}\right) \\
\text { s.t. } & (x, y, \mu, \phi, \chi, \eta) \text { solve }(31) \\
& u \in \mathcal{U} .
\end{aligned}
$$

Theorem 5.5. An equilibrium of the market with adjustable robust producers (29) and consumer (4) is given by $\left(\pi^{*}(\cdot), \eta^{*}, y^{*}, x^{*}(\cdot)\right)$.

Proof. We prove that $\left(\pi^{*}(\cdot), \eta^{*}, y^{*}, x^{*}(\cdot)\right)$ is an equilibrium by showing that no producer $j$ has an incentive to deviate from $y_{j}^{*}$ or $x_{j}^{*}(\cdot)$.

First, we show that given $\pi^{*}(\cdot), \eta^{*}$, and $y^{*}$, a producer $j$ with $y_{j}^{*}>0$ would not produce differently than $x_{j}^{*}$. Let $u$ be arbitrary but fixed and let $t \in \mathcal{T}$. From (31a), (31e), (31f), and the nonnegativity of $x^{*}, y^{*}, \mu^{*}$, and $\phi^{*}$ we obtain

$$
x_{j, t}^{*}=\left\{\begin{array}{lll}
y_{j}^{*} & \text { if } & p_{t}\left(\bar{x}_{t}^{*}\right)-c_{j, t}^{\operatorname{var}}(u)>0, \\
0 & \text { if } & p_{t}\left(\bar{x}_{t}^{*}\right)-c_{j, t}^{\operatorname{var}}(u)<0, \\
\operatorname{arb} . \in\left[0, y_{j}^{*}\right] & \text { if } & p_{t}\left(\bar{x}_{t}^{*}\right)-c_{j, t}^{\operatorname{var}}(u)=0 .
\end{array}\right.
$$

Since $\pi_{t}^{*}(u)=p_{t}\left(\bar{x}_{t}^{*}\right)$, this implies

$$
\left(\pi_{t}^{*}(u)-c_{j, t}^{\mathrm{var}}(u)\right) x_{j, t}^{\prime} \leq\left(\pi_{t}^{*}(u)-c_{j, t}^{\mathrm{var}}(u)\right) x_{j, t}^{*}
$$


for any $0 \leq x_{j}^{\prime} \leq y_{j}^{*}$ with $x_{j, t}^{\prime}(u) \neq x_{j, t}^{*}(u)$. Thus, by deviating from $x_{j}^{*}$, producer $j$ 's profit cannot be increased.

Next, we show that the worst case profit of every producer is zero. Clearly, this holds true for all producers with zero capacity. Let $i$ be a producer with $y_{i}^{*}>0$. Their objective value in scenario $u \in \mathcal{U}$, which we denote by $P_{i}^{*}(u)$, is given by

$$
P_{i}^{*}(u)=\sum_{t \in \mathcal{T}}\left(\left(\pi_{t}^{*}(u)-c_{i}^{\mathrm{var}}(u)\right) x_{i, t}^{*}\right)-\left(c_{i}^{\mathrm{inv}}-\eta_{i}^{*}\right) y_{i}^{*}
$$

From (34) it follows that $x_{i, t}^{*}$ is either 0 or $y_{i}^{*}$ or it does not influence the objective value. Thus, with $\mathcal{T}_{i, u}=\left\{t \in \mathcal{T}: p_{t}\left(\bar{x}_{t}^{*}\right)>c_{j, t}^{\operatorname{var}}(u)\right\}$, we have

$$
P_{i}^{*}(u)=\sum_{t \in \mathcal{T}_{i, u}}\left(\left(\pi_{t}^{*}(u)-c_{i}^{\mathrm{var}}(u)\right) y_{i}^{*}\right)-\left(c_{i}^{\mathrm{inv}}-\eta_{i}^{*}\right) y_{i}^{*}
$$

Thus, for the worst case profit we have

$$
\min _{u \in \mathcal{U}} P_{i}^{*}(u)=\min _{u \in \mathcal{U}}\left\{\sum_{t \in \mathcal{T}_{i, u}}\left(\left(\pi_{t}^{*}(u)-c_{i}^{\mathrm{var}}(u)\right) y_{i}^{*}\right)\right\}-\left(c_{i}^{\mathrm{inv}}-\eta_{i}^{*}\right) y_{i}^{*} .
$$

Inserting (33) from Lemma 5.4 for $\eta_{i}^{*}$ now yields

$$
\min _{u \in \mathcal{U}} P_{i}^{*}(u)=0 \text {. }
$$

Finally, we consider the case in which a producer $j$ deviates from $y_{j}^{*}$. First, let $u_{j}^{\min } \in \arg \min _{u \in \mathcal{U}} P_{j}^{*}(u)$. Now assume that producer $j$ invests in capacity $y_{j}^{\prime} \neq y_{j}^{*}$.

The profit of producer $j$ in $u_{j}^{\min }$ is given by

$$
\begin{aligned}
P_{j}^{\prime}\left(u_{j}^{\min }\right) & =\max _{0 \leq x_{j} \leq y_{j}^{\prime}}\left\{\sum_{t \in \mathcal{T}}\left(\left(\pi_{t}\left(u_{j}^{\min }\right)-c_{j}^{\mathrm{var}}\left(u_{j}^{\mathrm{min}}\right)\right) x_{j, t}\right)\right\}-\left(c_{j}^{\mathrm{inv}}-\eta_{j}^{*}\right) y_{j}^{\prime} \\
& =\sum_{t \in \mathcal{T}_{i, u}}\left(\left(\pi_{t}^{*}\left(u_{j}^{\min }\right)-c_{j}^{\mathrm{var}}\left(u_{j}^{\min }\right)\right) y_{j}^{\prime}\right)-\left(c_{j}^{\mathrm{inv}}-\eta_{j}^{*}\right) y_{j}^{\prime}
\end{aligned}
$$

Inserting (33) from Lemma 5.4 for $\eta_{j}^{*}$ yields $P_{j}^{\prime}\left(u_{j}^{\min }\right) \leq 0$. Since $P_{j}^{\prime}\left(u_{j}^{\text {min }}\right)$ is an upper bound for the worst case profit of producer $j$, this means that deviating from $y_{j}^{*}$ does not increase the worst case profit of producer $j$.

This proof concludes the section on robust peak load pricing. We showed how to compute equilibria in a market with strict robust producers and proved the possible existence of a gap to the corresponding robust central planner solutions. In the adjustable robust setting, we showed that the adjustable robust central planner cannot improve on the strict robust central planner. Finally, this last theorem showed how to offer an incentive via subsidies such that the equilibrium in the market with adjustable robust producers is equal to the robust central planner solution.

\section{Consequences for optimizing Risk measures}

Different approaches are known in optimization under uncertainty. Robust optimization is well-suited in cases where one has little knowledge about the shape and structure of the uncertainties and additionally the market participants are very risk averse. In cases where full information about the underlying uncertainties is available, e.g. if the probability distribution $F$ of the uncertain variables $u_{i, t} \in \mathcal{U}$ are known, and if the stochastic problem is algorithmically tractably, we could replace the robust optimization approach in (17) and (18) by a stochastic optimization 
problem. For (17) with the expectation instead of the worst-case realization in the objective, this would read

$$
\begin{aligned}
\min _{x, y \geq 0} & \sum_{i \in \mathcal{N}}\left(c_{i}^{\mathrm{inv}} y_{i}+\left(\sum_{t \in \mathcal{T}}\left(c_{i}^{\mathrm{var}}+a_{i, t} E\left(u_{i, t}\right)\right) x_{i, t}\right)\right) \\
\text { s.t. } & x_{i, t} \leq y_{i}, \quad i \in \mathcal{N}, t \in \mathcal{T} \\
& \sum_{i \in \mathcal{N}} x_{i, t}=d_{t}, \quad t \in \mathcal{T} .
\end{aligned}
$$

Here, the expectation essentially plays the role of a risk measure for which protection is sought by the (not very risk-averse) market participants. Still, there is a close connection to robust optimization. Indeed, it has been shown in e.g. Bertsimas and Brown [2009], that robust optimization can be used to analyze problems involving risk measures. With the techniques presented below, we can also exploit limited information on the (generally unknown) probability distribution $F: \mathbb{R}_{\geq 0}^{N} \rightarrow[0,1]$ of nonnegative random variables $u_{i}$ is available. The additional information on $F$ is going to inform the shape of the underlying uncertainty set $\mathcal{U}$ against which the producers and the central planner seek to protect themselves.

To capture the risk aversity of the market participants we rely on a concept that is widely considered in the economic literature - the value at risk (VaR). The value at risk at the confidence level $1-\alpha$ is the $(1-\alpha)$-quantile of a loss function $U \in \mathbb{R}$, i.e. a random variable $U$ that measures the loss of a market participant. Suppose that this loss function is distributed by $F_{U}$, then the value at risk at a confidence level of $1-0.05$ is $\operatorname{VaR}_{0.95}(U)=F_{U}^{-1}(0.95)$. In the following lines we omit $U$ and solely consider the (potentially unknown) distribution $F$. It is worth noting that information on the value at risk can be achieved without full knowledge on $F$. In particular, in El Ghaoui et al. [2003] the authors consider uncertain distributions and compute their worst-case value at risk without deriving the underlying distribution.

Here, we suppose that the value at risk is known and the central planner seeks to protect against this risk measure instead of e.g. the expected value considered in (35). As we deal with multivariate uncertainties we extend the above definition to a multivariate value at risk (MVaR) defined by Prékopa (Prékopa [1990] for discrete probability distributions, Prékopa [2012] for continuous distributions):

$$
\operatorname{MVaR}_{1-\alpha}:=\left\{u \in \mathbb{R}^{N}: F(u)=1-\alpha\right\} .
$$

Since our random variables are nonnegative, a natural definition of the global uncertainty set $\mathcal{U}$ is

$$
\mathcal{U}:=\left\{u \in \mathbb{R}_{\geq 0}^{N}: F(u) \leq 1-\alpha\right\} .
$$

As a probability distribution, $F$ is monotonously increasing. Hence, on the one hand it is quasiconvex implying the convexity of its sublevel set $\left\{u \in \mathbb{R}^{N}: F(u) \leq 1-\alpha\right\}$ and consequently the convexity of $\mathcal{U}$. On the other hand the monotonicity restricts the $u_{i}$ from above and implies compactness of $\mathcal{U}$. Moreover, we can ensure that its projections on the unit vectors $e_{i}$ form the interval $[0,1]$ by rescaling $\mathcal{U}$ to $\operatorname{diag}\left(\operatorname{VaR}_{1-\alpha}^{1}, \ldots, \operatorname{VaR}_{1-\alpha}^{N}\right)^{-1} \mathcal{U}$ and thus have established the necessary conditions needed to directly apply the theory above for a predefined uncertainty $\operatorname{set} \mathcal{U}=\{u \in$ $\left.\mathbb{R}_{\geq 0}^{N}: u \leq \mathrm{MVaR}_{1-\alpha}\right\}$.

The following paragraphs illustrate this at the example of the robust peak load pricing with fixed demand from Section 4. Here, we let the random part of the variable costs $u$ be distributed according to the distribution $F$. Hence, the $u_{i}$ are distributed with respect to the marginal distributions $F_{i}$ and the producers aim to optimize the worst case uncertainty in $\mathcal{U}_{i}=\left\{u_{i} \in \mathbb{R}_{\geq 0}: F_{i}\left(u_{i}\right) \leq 1-\alpha\right\}$, which we 
will denote (in slight abuse of notation) as the marginal value at risk $\operatorname{VaR}_{1-\alpha}^{i}$. We obtain the following variant of (16):

$$
\begin{aligned}
\max _{x_{i}, y_{i} \geq 0} & \sum_{t \in \mathcal{T}}\left(\left(\pi_{t}-\left(c_{i}^{\mathrm{var}}+\mathrm{VaR}_{1-\alpha}^{i}\right)\right) x_{i, t}\right)-c_{i}^{\mathrm{inv}} y_{i} \\
\text { s.t. } & x_{i, t} \leq y_{i}, \quad t \in \mathcal{T} .
\end{aligned}
$$

Observe that the rescaled $\mathcal{U}$ satisfies our necessary assumptions and setting $a_{i}=$ $\mathrm{VaR}_{1-\alpha}^{i}$ negates the rescaling effect on the definitions of the worst case total cost

$$
E_{R}=\max _{u \in \mathcal{U}} \sum_{i \in \mathcal{N}}\left(c_{i}^{\mathrm{inv}} y_{i}^{*}+\left(\sum_{t \in \mathcal{T}}\left(c_{i}^{\mathrm{var}}+u_{i, t}\right) x_{i, t}^{*}\right)\right)
$$

of a market equilibrium $\left(x^{*}, y^{*}\right)$ in $(17)$ and the corresponding robust central planner problem

$$
\begin{aligned}
C_{R}=\min _{x, y \geq 0} & \max _{u \in \mathcal{U}} \sum_{i \in \mathcal{N}}\left(c_{i}^{\mathrm{inv}} y_{i}+\left(\sum_{t \in \mathcal{T}}\left(c_{i}^{\mathrm{var}}+u_{i, t}\right) x_{i, t}\right)\right) \\
\text { s.t. } & x_{i, t} \leq y_{i}, \quad i \in \mathcal{N}, t \in \mathcal{T}, \\
& \sum_{i \in \mathcal{N}} x_{i, t}=d_{t}, \quad t \in \mathcal{T} .
\end{aligned}
$$

Thus, we obtain the following corollary of Theorem 4.2 :

Corollary 6.1. Let $\mathcal{U}=\left\{u \in \mathbb{R}_{\geq 0}^{N}: u \leq \mathrm{MVaR}_{1-\alpha}\right\}$. Then the following inequalities are satisfied

$$
C_{R} \leq E_{R} \leq \frac{1}{\tau\left(\operatorname{diag}\left(\mathrm{VaR}_{1-\alpha}^{1}, \ldots, \mathrm{VaR}_{1-\alpha}^{N}\right)^{-1} \mathcal{U}\right)} C_{R}
$$

We note that although the scaling matrix $\left(\operatorname{diag}\left(\operatorname{VaR}_{1-\alpha}^{1}, \ldots, \operatorname{VaR}_{1-\alpha}^{N}\right)^{-1}\right.$ and $\mathrm{MVaR}_{1-\alpha}$ share the same underlying distribution it is not trivial to simplify the above result by exploiting this relationship. We further add that similar corollaries can also be obtained for the other results in Sections 4 and 5 .

One major criticism of the value at risk is that it is, although widely used, not a coherent risk measure, i.e. it lacks the desirable properties derived by Artzner Artzner et al. [1999]. However, for nonnegative random variables also coherent risk measures can partly be included in our theory as they are related to (distributionally) robust optimization through the following statement (see Bertsimas and Thiele [2014] or Theorem 3.1.1 in Brown [2006]):

A risk measure $\mu: \mathcal{X} \rightarrow \mathbb{R}$ is coherent if and only if there exists a family of probability measures $\mathcal{Q}$ such that

$$
\mu(X)=\sup _{q \in \mathcal{Q}} E_{q}(X) \text { for every random variable } X \in \mathcal{X},
$$

where $E_{q}$ denotes the expected value with respect to the probability distribution $q$. Thus, suppose the producers aim to optimize their respective risk measures $\mu_{i}: \mathbb{R}_{\geq 0} \rightarrow \mathbb{R}$ acting on the $i$-th component of the random vector $u \in[0,1]^{N}$, whereas the central planner aims to optimize a risk measure $\mu: \mathbb{R}_{\geq 0}^{N} \rightarrow \mathbb{R}$ acting on the whole random vector $u$. It is natural to assume that the choice of the risk measures and its underlying set $\mathcal{Q}$ is data driven from a set of past realizations of uncertainty $\mathcal{A}=\left\{\hat{u}^{1}, \ldots, \hat{u}^{K}\right\}$. Similarly, as we have done for the value at risk, our goal for the remainder of this section is to follow the arguments in Brown [2006] to link the peak load pricing with respect to the risk measures $\mu$ to our results from Sections 4 and 5 by determining a convenient uncertainty set $\mathcal{U}$.

For a fair comparison we assume that both, the central planner and the producer evaluate their risk based on the same set of probability distributions $\mathcal{Q}$ that is supported solely on $\mathcal{A}$ (see Assumption 3.2.1 in Brown [2006] or Bertsimas and Thiele 
[2014]). In addition we assume $\hat{u}^{1}=0$. Hence, instead of (16), the producers aim to optimize

$$
\begin{aligned}
\max _{x_{i}, y_{i} \geq 0} & \sum_{t \in \mathcal{T}}\left(\left(\pi_{t}-\left(c_{i}^{\mathrm{var}}+\mu_{i}\left(u_{i}\right)\right)\right) x_{i, t}\right)-c_{i}^{\mathrm{inv}} y_{i} \\
\text { s.t. } & x_{i, t} \leq y_{i}, \quad t \in \mathcal{T},
\end{aligned}
$$

which according to Theorem 3.2.1 in Brown [2006] is equivalent to

$$
\begin{aligned}
\max _{x_{i}, y_{i} \geq 0} \min _{u_{i} \in \mathcal{U}_{i}} & \sum_{t \in \mathcal{T}}\left(\left(\pi_{t}-\left(c_{i}^{\mathrm{var}}+u_{i}\right)\right) x_{i, t}\right)-c_{i}^{\text {inv }} y_{i} \\
\text { s.t. } & x_{i, t} \leq y_{i}, \quad t \in \mathcal{T},
\end{aligned}
$$

where, since $\hat{u}^{1}=0$, the considered $\mathcal{U}_{i}$ are intervals that satisfy

$$
\begin{aligned}
\mathcal{U}_{i} & =\operatorname{conv}\left(\sum_{j=1}^{K} q_{j} \hat{u}_{i}^{j}: q \in \mathcal{Q}\right)=\left[0, \max _{q \in \mathcal{Q}} \sum_{j=1}^{K} q_{j} \hat{u}_{i}^{j}\right] \\
& \subseteq \operatorname{conv}\left(\left\{\hat{u}_{i}^{1}, \ldots, \hat{u}_{i}^{K}\right\}\right)=\left[0, \max _{j=1, \ldots, K} \hat{u}_{i}^{j}\right] .
\end{aligned}
$$

Moreover, if we denote $m_{i}:=\max _{q \in \mathcal{Q}} \sum_{j=1}^{K} q_{j} \hat{u}_{i}^{j}$, we can apply the same rescaling trick as we did for the value at risk and obtain an uncertainty set

$$
\mathcal{U}=\operatorname{diag}\left(\frac{1}{m_{1}}, \ldots, \frac{1}{m_{N}}\right) \operatorname{conv}\left(\sum_{j=1}^{K} q_{j} \hat{u}^{j}: q \in \mathcal{Q}\right)
$$

that satisfies the necessary conditions at the beginning of Section 4, i.e., it is a polytope and its projection on any coordinate axis is $[0,1]$. Thus, we obtain the following similar corollary of Theorem 4.2:

Corollary 6.2. Given a set of probability distributions $\mathcal{Q}$ and the uncertainty set $\mathcal{U}=\operatorname{conv}\left(\sum_{j=1}^{K} q_{j} \hat{u}^{j}: q \in \mathcal{Q}\right)$, then the following inequalities are satisfied

$$
C_{R} \leq E_{R} \leq \frac{1}{\tau\left(\operatorname{diag}\left(\frac{1}{m_{1}}, \ldots, \frac{1}{m_{N}}\right) \mathcal{U}\right)} C_{R} .
$$

Again, similar corollaries can also be derived for the other results in Sections 4 and 5 as the key to these applications is simply to construct a valid uncertainty set $\mathcal{U}$. Although a further investigation into the connections of risk measures to our uncertainty sets appears to be very promising, it exceeds the scope of the present article and is thus postponed to future research. To summarize, after having obtained structural insights for both single-stage and two-stage robust market problems under uncertain cost together with the corresponding implications for the value at risk, the subsequent section concludes this work with a brief summary of the results obtained here.

\section{Conclusion}

In the present article, we considered equilibrium problems where firms in a market context maximize their profits in a robust way when selling their output. Our analysis brings the robust optimization perspective into the context of equilibrium problems. In our setup we first considered the single-stage or non-adjustable robust setting where firms can sell their output. We then went one step further and studied the more complex adjustable case where a part of the variables are wait-and-see decisions. We compared equilibrium outcomes with the corresponding centralized robust optimization problem where the sum of all profits is maximized. 
We established existence of the resulting robust equilibrium problems and also determined the solution of the corresponding robust central planner. We showed that the market equilibrium for the perfectly competitive firms differs from the solution of the centralized optimization problem. For the different scenarios considered we furthermore are able to determine the resulting price of anarchy. In the case of non-adjustable robustness, for fixed demand in every time step the price of anarchy is bounded. It is unbounded if market demands for the different time periods are modelled to be elastic. As a direct application of the results we considered settings where the market participants aim to optimize their respective risk measures, instead of their worst-case production costs. The risk measures of the market participants then determine the uncertainty set for our robust problem. We could show that all our results can be directly applied to these settings. In sum, our analysis thus provides first important insights on bringing robust optimization approaches in the context of market equilibrium problems.

\section{ACKNowledgments}

We are grateful to Veronika Grimm for stimulating discussions. This research has been performed as part of the Energie Campus Nürnberg (EnCN) and is supported by funding of the Bavarian State Government. The authors thank the Deutsche Forschungsgemeinschaft for their support within projects B06, B07 and B09 in the Sonderforschungsbereich/Transregio 154 "Mathematical Modelling, Simulation and Optimization using the Example of Gas Networks".

\section{DECLARATIONS OF INTEREST}

None

\section{REFERENCES}

Michele Aghassi and Dimitris Bertsimas. Robust game theory. Mathematical Programming, 107:231-273, 2006. doi: 10.1007/s10107-005-0686-0.

Philippe Artzner, Freddy Delbaen, Jean-Marc Eber, and David Heath. Coherent measures of risk. Mathematical Finance, 9(3):203-228, 1999. doi: 10.1111/1467-9965.00068.

A. Ben-Tal, L. El Ghaoui, and A. Nemirovski. Robust Optimization. Princeton University Press, 2009. ISBN 9781400831050.

Dimitris Bertsimas and David B. Brown. Constructing uncertainty sets for robust linear optimization. Operations Research, 57(6):1483-1495, 2009. doi: 10.1287/ opre.1080.0646.

Dimitris Bertsimas and Aurélie Thiele. Robust and data-driven optimization: Modern decision making under uncertainty. INFORMS TutORials in Operations Research, pages 95-122, 2014. doi: 10.1287/educ.1063.0022.

Christian Biefel, Frauke Liers, Jan Rolfes, and Martin Schmidt. Affinely adjustable robust linear complementarity problems. SIAM Journal on Optimization, 32: 152-172, 2022. doi: 10.1137/20M1359778.

John R. Birge and Francois Louveaux. Introduction to Stochastic Programming. Springer Science \& Business Media, 2011. doi: 10.1007/978-1-4614-0237-4.

D. Brown. Risk and robust optimization, 2006 URL http://dspace.mit.edu/handle/1721.1/37894.

Emre Çelebi, Vanessa Krebs, and Martin Schmidt. $\gamma$-robust electricity market equilibrium models with transmission and generation investments. Energy Systems, pages 1-28, 2021. doi: 10.1007/s12667-020-00411-x.

Michael A Crew, Chitru S Fernando, and Paul R Kleindorfer. The theory of peakload pricing: A survey. Journal of regulatory economics, 8(3):215-248, 1995. 
Arup Dey and Kais Zaman. A robust optimization approach for solving two-person games under interval uncertainty. Computers $\&$ Operations Research, 119:104937, 2020. ISSN 0305-0548. doi: 10.1016/j.cor.2020.104937.

Pradeep Dubey. Inefficiency of Nash equilibria. Math. Oper. Res., 11(1):1-8, 1986. ISSN 0364-765X. doi: 10.1287/moor.11.1.1.

Andreas Ehrenmann and Yves Smeers. Generation capacity expansion in a risky environment: A stochastic equilibrium analysis. Operations Research, 59:13321346, 12 2011. doi: 10.2307/41316039.

Laurent El Ghaoui, Maksim Oks, and Francois Oustry. Worst-case value-at-risk and robust portfolio optimization: A conic programming approach. Operations Research, 51(4):543-556, 2003. doi: 10.1287/opre.51.4.543.16101.

Bruno Fanzeres, Shabbir Ahmed, and Alexandre Street. Robust strategic bidding in auction-based markets. European Journal of Operational Research, 272(3): 1158-1172, 2019. ISSN 0377-2217. doi: 10.1016/j.ejor.2018.07.027.

Henri Gérard, Vincent Leclère, and Andy Philpott. On risk averse competitive equilibrium. Operations Research Letters, 46(1):19-26, 2018. ISSN 0167-6377. doi: $10.1016 /$ j.orl.2017.10.011.

Paul Joskow and Jean Tirole. Reliability and competitive electricity markets. The RAND Journal of Economics, 38(1):60-84, 2007. doi: 10.1111/j.1756-2171.2007. tb00044.x.

Elias Koutsoupias and Christos Papadimitriou. Worst-case equilibria. In STACS 99 (Trier), volume 1563 of Lecture Notes in Comput. Sci., pages 404-413. Springer, Berlin, 1999. doi: 10.1007/3-540-49116-3_38.

Anja Kramer, Vanessa Krebs, and Martin Schmidt. Strictly and $\gamma$ robust counterparts of electricity market models: Perfect competition and nash-cournot equilibria. Technical report, 2018. URL http: //www .optimization-online.org/DB_HTML/2018/07/6709.html.

Vanessa Krebs and Martin Schmidt. $\gamma$-robust linear complementarity problems. Optimization Methods and Software, pages 1-34, 2020. doi: 10.1080/10556788. 2020.1825708.

Vanessa Krebs, Michael Müller, and Martin Schmidt. $\gamma$-robust linear complementarity problems with ellipsoidal uncertainty sets. International Transactions in Operational Research, 2021. doi: 10.1111/itor.12988.

Andreu Mas-Colell, Michael D. Whinston, and Jerry R. Green. Microeconomic Theory. Oxford University Press, New York, 1995a.

Andreu Mas-Colell, Michael D. Whinston, and Jerry R. Green. Microeconomic Theory. Oxford University Press, 1995b.

Frederic H. Murphy and Yves Smeers. Generation capacity expansion in imperfectly competitive restructured electricity markets. Operations Research, 53(4):646-661, 2005. doi: 10.1287/opre.1050.0211.

Richard P O'Neill, Paul M Sotkiewicz, Benjamin F Hobbs, Michael H Rothkopf, and William R Stewart Jr. Efficient market-clearing prices in markets with nonconvexities. European Journal of Operational Research, 164(1):269-285, 2005.

Jong-Shi Pang, Suvrajeet Sen, and Uday Shanbhag. Two-stage non-cooperative games with risk-averse players. Mathematical Programming, 165, 04 2017. doi: 10.1007/s10107-017-1148-1.

A. Philpott, Michael Ferris, and Roger Wets. Equilibrium, uncertainty and risk in hydro-thermal electricity systems. Mathematical Programming, 157:483-513, 01 2016. doi: 10.1007/s10107-015-0972-4.

András Prékopa. Dual method for the solution of a one-stage stochastic programming problem with random RHS obeying a discrete probability distribution. ZOR Methods Model. Oper. Res., 34(6):441-461, 1990. doi: 10.1007/BF01421551. 
András Prékopa. Multivariate value at risk and related topics. Ann. Oper. Res., 193(1):49-69, 2012. doi: 10.1007/s10479-010-0790-2.

Daniel Ralph and Yves Smeers. Risk trading and endogenous probabilities in investment equilibria. SIAM Journal on Optimization, 25:2589-2611, 10 2015. doi: $10.1137 / 110851778$.

Lars Schewe and Martin Schmidt. Optimierung von Versorgungsnetzen: Mathematische Modellierung und Lösungstechniken. Springer-Verlag, 2019.

Dane Schiro, Benjamin Hobbs, and Jong-Shi Pang. Perfectly competitive capacity expansion games with risk-averse participants. Computational Optimization and Applications, 65, 11 2016. doi: 10.1007/s10589-015-9798-5.

Hoang Tuy. Convex Analysis and Global Optimization. Springer, 1998.

Ming Wang, Kin Keung Lai, Stephen C. H. Leung, and Ning Shi. A robust optimization model for dynamic market with uncertain production cost. 61(2):187-207, 2012. ISSN 0233-1934. doi: 10.1080/02331934.2010.537338.

Dan Wu, Ji-Ye Han, and Jing-Hao Zhu. Robust solutions to uncertain linear complementarity problems. Acta Mathematicae Applicatae Sinica, English Series, 27 (2):339-352, 2011. doi: 10.1007/s10255-010-0033-y.

Yue Xie and Uday V. Shanbhag. On robust solutions to uncertain linear complementarity problems and their variants. SIAM Journal on Optimization, 26(4): 2120-2159, 2016. doi: 10.1137/15M1010427.

Gregor Zöttl. A framework of peak load pricing with strategic firms. Operations Research, 58:1637-1649, 12 2010. doi: 10.1287/opre.1100.0836.

(C. Biefel, F. Liers, J. Rolfes) Friedrich-Alexander-Universität Erlangen-Nürnberg, Germany, Department of Data Science, Cauerstr. 11, 91058 Erlangen; Energie CamPus Nürnberg, Fürther Str. 250, 90429 Nürnberg, Germany

Email address: \{christian.biefel,frauke.liers, jan.rolfes\}@fau.de

(L. Schewe) University of Edinburgh, School of Mathematics and Maxwell Institute for Mathematical Sciences, James Clerk Maxwell Building, Peter Guthrie Tait Road, Edinburgh, EH9 3FD, UK; Energie Campus Nürnberg, Fürther Str. 250, 90429 Nürnberg, GERMANY

Email address: lars.schewe@ed.ac.uk

(G. Zöttl) Friedrich-Alexander-Universität Erlangen-Nürnberg, Germany, Institute of Economic Research, Lange Gasse 20, 90403 Nürnberg; Energie Campus Nürnberg, Fürther Str. 250, 90429 NürnberG, GERMAnY

Email address: gregor.zoettl@fau.de 